\title{
Showcasting the use of big data for policy purposes
}

\section{Per Nymand-Andesen}

Adviser, Director General Statistics, European Central Bank.

"Progress lies not in enhancing what is, but in advancing towards what will be" (Khalin Gibran).

\begin{abstract}
While the availability and accessibility of large data sources is a rich field for statisticians, economists, econometricians and forecasters, it has been relatively unexploited for producing substainable and reliable statistics for policy purposes. It is of particular interest if such large sources could help to detect trends and turning points within the economy, thereby providing supplementary and more timely information compared to the "traditional" toolkit of policy makers. These supplementary statistics may provide further insights contributing to guiding policy actions as well as to assessing the subsequent impact and associated risks of these policy decisions on the financial system and real economy. Big data sources could assist policy makers in obtaining a nearly real-time snapshot of the economy as well as providing early warning indicators. This may be particularly welcome in the light of the shortcomings observed in the run-up to the financial crisis. In particular regulators have been keen to expand the data collection so as to better monitor financial intermediaries, financial risks and vulnerabilities.

The way forward may therefore be to develop and apply a structural approach for piloting the use of big data. While it may be reasonable to expect that "big data" suppliers have different business and data models, it is important to assess their usefulness according to a standardised set of five key criteria following a production cycle; "Input", "Quality”, "Production”, "Results" and "Assessment" as part of exploring its relevance for producing substainable and reliable statistics for policy making purposes.
\end{abstract}

Keywords: Policy making; big data; statistics. 